We believe ${ }^{1}$ that some if not most cases of this condition belong to the group of collagen disorders, many probably being connected in some way with rheumatoid disease. We are unconvinced by the arguments advanced that an alteration of nomenclature at this juncture for a condition whose aetiology remains obscure is likely to be of any advantage.-We are, etc.,

RICHARD WARD.

RANDAL STALKER.

Royal Infirmary,

REFERENCE

${ }^{1}$ Ward, R., and Stalker, R., Lancet, 1964, 2, 477

\section{Oral Contraceptives and Breast Cancer}

SIR,-May I reply to Mr. J. J. Shipman's letter (5 September, p. 629) ? Referring to the use of oral contraceptives in the presence of carcinoma of the breast, I would not agree that their use should be " discontinued if carcinoma of the breast is thought or known to be present." Consideration must be given to the possible outcome of not taking the contraceptive drug. The obvious risk is pregnancy, and pregnancy is known to have a serious effect on the progress of some breast carcinomas. The oral contraceptives are in high demand because they have practical application. Other forms of contraceptive fail only too frequently because a natural urge and an available contraceptive do not coincide. The need to avoid pregnancy should be considered against the possible effect of hormones administered orally on a carcinoma of breast, and I believe that the risks of pregnancy are greater.

An alternative method of management is by sterilization associated with bilateral salpingooophorectomy, but few people accept this as a realistic approach.-I am, etc.,

Bangor, Carns.

GARETh Lloyd.

\section{Protection Against Tetanus}

SIR,-In the valuable and concise statement by the Ministry of Health giving the recommendations of the Advisory Group on prutection against tetanus (25 July, p. 243) there is one point which would seem to need clarification. It rather infers that when antitoxin is given an injection of adsorbed tetanus toxoid should be given simultaneously. Can one be certain that the passive immunity thus procured will not interfere with the development of active immunization? Would it not be better to arrange for a course of tetanus toxoid to commence, say, one month later?

Perhaps Dr. H. J. Parish or one of the other authorities would clear up this point, about which some of us are, are present, in doubt.-I am, etc.,

London N.W.7.

A. H. Morley.

\section{Prophylactic Eye-drops}

SIR,-In the B.M.F. (4 July, p. 47) there was an article on failure to control the venereal diseases. In the same issue (Obstetrics in General Practice-Care of the
Newborn Infant, p. 36) under Care of the Eyes I read: "Unless there is a maternal history of vaginal discharge during pregnancy or evidence of discharge during labour, routine prophylactic eye-drops are unnecessary."

I hope Professor Gaisford will think again on this statement. Surely it is a recognized fact to-day that many patients suffering from either syphilis or gonorrhoea, whether male or female, show no obvious cliniçal signs and do not complain of symptoms. At the V.D. clinics I used to see a considerable percentage of cases of gonorrhoea in women who had no symptoms and who showed no obvious clinical signs and yet films or cultures from cervix, urethra, or rectum showed gonococci. The same applies to streptococci and coliform bacilli. I would here like to emphasize the importance of cultures : gonococci can frequently be found in cultures and not in films, and I fear that many laboratories are not very expert in this investigation.

At the Birmingham Eye Hospital, which I used to attend, the number of cases of ophthalmia neonatorum was often in the region of 700 per annum, only a small percentage being gonococcal. I suggest that this considerable number is due either to lack of prophylactic eye-drops or to the failure to instil the drops into the conjunctival sac properly, as I cannot remember a single case of ophthalmia neonatorum occurring in the V.D. maternity ward, where we dealt with some 50 or more midwifery cases a year and where eye-drops were used as routine.

It is, of course, essential that the drops should be non-irritating as well as antiseptic. In any event I cannot see that the eye-drops can do any harm, and in my opinion they should always be used in case there is infection.

Blindness and corneal opacity caused by ophthalmia neonatorum is a tragedy, and if it can be prevented by the simple instillation of a few drops at the time of birth I contend that this should always be done.-I am, etc.,

\section{Stratford-on-Avon, \\ ERIC Assinder. Warwickshire.}

\section{Is Arterial Puncture Dangerous?}

SIR,-In reply to Professor W. W. Mushin's letter (1 August, p. 310), I should like to instance three complications that I have seen following arterial puncture in the last three years.

Firstly, a baby of 9 months, who very nearly bled to death after a femoral artery puncture for cardiac catheterization. The bleeding was discovered in this instance some half an hour after the child had been returned to its cot, ostensibly well, following its cardiac catheterization and left ventriculogram.

The second instance is of a middle-aged lady with valvular disease of the heart, who had a femoral-artery puncture for a left ventriculogram. She subsequently developed a haematoma immediately after the operation, which in the next three weeks grew in size, unchecked, to such an extent that the skin overlying a part of the haematoma became gangrenous. The haematoma itself became infected, and the femoral artery below the haematoma burst, very nearly causing loss of the patient's life. It will be realized that with a massive haematoma in the groin, as in this instance, control of the femoral artery after it had burst was exceedingly difficult.

Thirdly, it is common knowledge that the femoral-artery puncture in small children is frequently accompanied by arterial spasm, and I have seen this last with blanching of the leg for upwards of 25-30 minutes.

Bleeding, following puncture of a highpressure vessel, can and does occur, but I am sure that this complication could be numerically reduced by greater vigilance, less haste, and limitation of the technique to fewer persons. I would be interested to hear of any arterial spasm in babies, of sufficient duration to cause anxiety with regard to limb ischaemia.-I am, etc.,

Queen Elizabeth Hospital,
Birmingham 15.

BRYAN WALDRON.

\section{Early Discharge of Maternity Patients}

SIR,-With reference to the leading article on the early discharge of maternity patients (11 July, p. 70) we would like to offer for comparison the obstetrical statistics of Brookhaven Memorial Hospital, Patchogue, New York. From July 1963 to June 19641,947 deliveries were performed in the 30-bed maternity suite. This is a turnover of approximately 65 patients per bed per annum. The average length of stay was 3.74 days. The average length of stay of vaginal deliveries was 3.48 days; the average length of stay for caesarean deliveries was 7.6 days. There was a total of 109 caesarean deliveries. This length of hospital confinement is about the average for this part of the United States. It should be noted the cases are completely unselected and that all deliveries are performed in hospitals, not in patients' homes.

Although the Obstetrical Flying Squad was a good thing in its day, it is now completely out-dated. The emergencies dealt with by such a team could have been handled much more efficiently if the patient had been in the hospital during her labour. By using beds realistically room can be made to accommodate the mothers who, because of shortage of space, are forced to accept delivery at home.

It should be realized that the hospital is the place for all deliveries, and that domiciliary obstetrics is now completely out-dated. However, it should not be forgotten that the purpose of the hospital is to provide medical care, not hotel accommodation.-We are, etc.,

MARKS G. JACOBY.

James Russell Clark.

Brookhaven Memorial Hospital,

Patchogue, N.Y., U.S.A.

\section{Hazards of Groundnuts}

SIR,-The reccgnition of a new disease responsible for the dcath of many turkeys in $1960^{1}$; the discovery that this resulted from the consumption of groundnut meal contaminated with the fungus Aspergillus flavus, ${ }^{2}$ and the establishment that aflatoxin from this fungus caused carcinoma of the liver in rats $^{3}$ in 1961 ; the detailed study of the chemistry of aflatoxin ${ }^{4}$ and the dissemination of criteria to help exclude infected groundnuts from animal feeds ${ }^{5}$ in 1962; and finally the elucidation of the structure of the two major toxic metabolites ${ }^{8}$ and the 
production of sarcomata by the subcutaneous injection of the toxin in rats in $1963^{7}$ have all been scientific achievements of the highest importance. However, these successes have as yet had no effect on reducing the possible hazards to health of this toxin on the inhabitants of developing countries.

The part of this research not directed to the furtherance of pure science has been mainly geared to ensure (1) that all animal feeds made from groundnuts are non-toxic, (2) that toxic reactions do not occur in humans as a result of consumption either of imported groundnuts or their products, and (3) that this fungus has minimal effects on commerce in groundnuts.

These are worthwhile considerations, but as was emphasized at the Sixth International Congress of Nutrition in $1963^{8}$ and as you have pointed out in a recent editorial ( 25 July, p. 204) the problem may be especially important for the many tropical countries where the consumption of groundnuts is high.

In the annual report of the Nutrition Unit of the Ministry of Health, Dar es Salaam, Tanganyika, for 1962 , I wrote, "This year East African groundnuts were found to be infected by this fungus ( $A$. flavus), and certain groundnut products were withdrawn from the market. Despite the fact that no toxic effects have been proved to occur in man it seemed worth looking into this question in view of our policy to encourage the greater consumption of groundnuts. It seemed not beyond the bounds of pcssibility that the relatively high incidence $\mathrm{rf}$ primary carcinoma of the liver here compared with Europe and North America might stem from this toxic cause." An investigation was therefore undertaken to see if a correlation existed between areas of high groundnut consumption and of high incidence of primary liver cancer in Tanganyika. The figures obtained were, however, not large enough to be statistically significant.

Besides primary liver carcinoma the possibility that liver fibrosis, a condition commonly encountered in Tanganyika, might also result from a prolonged consumption of aflatoxin in groundnuts was investigated. Liver-function tests were performed on a group of regular groundnut eaters in 1962 and they showed a high incidence of abnormal livers. However, in 1963 liverfunction tests carried out on a group of prisoners who from childhood had consumed large quantities of groundnuts and on a control group who seldom ate groundnuts showed no significant difference in the incidence of liver disease between the two groups. The numbers of subjects involved were small, and I do not regard the results of this work as being in any way conclusive. All were based on the assumption that a fair proportion of all groundnuts eaten would be infected with this common mould.

I wish, however, to stress that, though the laboratory research mentioned in the first paragraph of this letter is of extreme importance, there is an urgent need for the investigation of the possible toxic effects of the aflatoxin being daily consumed by millions of people in three continents. This requires epidemiological studies, rather diffcult field investigations, and the type of geographical research which our national research organizations are often unable and unwilling to undertake. Does this not provide a good example and reason for supporting a W.H.O. Research Centre? Would such a centre not have been able in 1962 to present us with maps showing areas of high groundnut consumption, and related this to the geographical distribution of fibrosis and primary carcinoma of the iiver? Would it not now be known whether Indian women in Brazil and Wagogo women in Africa secrete aflatoxin in their breast milk ? This knowledge might be more important than knowing that cattle and guinea-pigs do. Would we not have a report on the effects of aflatoxin on the already diseased livers of the very large numbers of children suffering from kwashiorker?

If in $1962 \mathrm{I}$ and my colleagues in Tanganyika had had such a research centre in Edinburgh to turn to we would have been very happy.-I am, etc.,

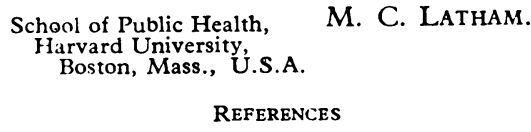

\section{"An Outline of Psychiatry"}

SIR,-I would like to point out that Dr. D. W. Kay in his review of An Outline of Psychiatry in the B.M.F. (29 August, p. 559) misrepresents my book and makes quite unjustified criticisms of it.

Thus, in my book I write: "I believe that in any given case all factors which may possibly be relevant should be considered, and the appropriate measures based on empirical knowledge, psycho-analytic theory, sociology or common sense, should be applied."

Dr. Kay quotes part of this statement, but omits the key words "based on empirical knowledge, psycho-analytic theory, sociology or common sense," and then accuses me of assuming that treatment follows logically from considerations of aetiology. He goes on to chide me for not producing a coherent theory of psychiatric aetiology and symptomatology. As no such theory exists, I believe that to fill a textbook with idle speculation would be a disservice to the teaching of psychiatry.

Kay also complains that my description of symptoms sometimes obscures the illness as a whole. $\mathrm{He}$ supports this by quoting examples from a chapter on symptomatology in which I attempt to define psychiatric symptoms and signs and to discuss their diagnostic significance. That this is needed can easily be seen from the most casual perusal of psychiatric literature in English. For example, two academic British psychiatrists have recently made the following inaccurate statement: "The presence of primary delusions is in general regarded as unequivocal evidence of schizophrenia proper." It is well known in the German literature ${ }^{2}$ that one common type of primary delusion is not diagnostic of schizophrenia. This point is made clear in my book.-I am, etc.,

Frank Fish.

Department of Psychological Medicine, niversity of Edinburgh, Edinburgh 8.

\section{REFERENCES}

1 Roth, M., and Kay, D. W. K., f. ment. Sci., $1962,108,118$.

Schneider, K., Clinical Psychopathology (trans. Hamilton, M. W.), 1959. Grune and Stratton, New York.

\section{Hierarchies in Mental Hospitals}

SIR,-It is a little odd that Dr. C. Entwistle (5 September, p. 631), if he is writing of his own position, does not appreciate his. rights as a consultant psychiatrist, which are the same as those of a consultant in any branch of medicine; if he does not exercise them much of the fault must be laid at his own door. Nor does he seem to be aware that when the post of medical superintendent of a mental hospital falls vacant the hospital management committee is asked by its board whether it wishes to have the post filled or not. To judge by your advertisement columns, many hospital management committees obviously do not agree with $\mathrm{Dr}$, Entwistle. Nor does the Ministry of Health, which begins a recent circular (June 1964) entitled "Improving the Effectiveness of Hospitals for the Mentally IIl" with the words: "There are many examples in the history of psychiatry of mental hospitals that were, in their time, outstanding. In most cases the achievements of these hospitals were the result of the enthusiasm either of one individual, usually the medical superintendent, or a group of medical staff."I am, etc.,

St. Lawrence's Hospital,
Caterham, Surrey.

JoHN Gibson.

\section{Drugs for Depression}

SIR,--In your leading article on "Drugs for Depression" (29 August, p. 522) you properly mention the "notable lack of any generally acceptable classification of depression." You go on to say that from "the practical point of view and in order to avoid mistakes in treatment it is important to have a clear classification." With this few would disagree, but it is, I submit, misleading to state so emphatically that there are " two different types of depression-reactive and endogenous," which you then proceed to describe. If differentiation were as simple as you suggest disagreement over classification would scarcely exist.

It is true that in Great Britain in recent years evidence has been collected which suggests that the depressive illness can be roughly divided into two groups, variously labelled. This evidence is summarized by Kiloh and Garside ${ }^{1}$ in a paper in which they present supportive data based on a factorial analysis of symptoms. Like others, they admit, however, that many cases cannot easily be classified in either group. Moreover, recent American factorial studies ${ }^{2}$ extract three or four factors, which suggest that more than two types of depression exist.

At Whitchurch Hospital recently we have given a symptom questionarys to 118 cases 